\title{
Basales, prandiales oder Mischinsulin: Welches Regime ist das Beste?
}

Hintergrund: Bisher konnte für keines der bekannten Insulinregime eine Überlegenheit eindeutig nachgewiesen werden.

Methoden: In die 3-jährige, offene, multizentrische „Treating to Target in Type 2 Diabetes“ (4-T)-Studie wurden 708 Typ-2-Diabetiker mit unbefriedigender Stoffwechsellage unter maximal tolerierter Dosis von Metformin und Sulfonylharnstoff eingeschlossen. Randomisiert erhielten die Teilnehmer entweder zweimal täglich Mischinsulin (inklusive Insulin Aspart), prandiales Insulin Aspart dreimal täglich oder einbzw. zweimal täglich das langwirksame Insulinanalogon Detemir. Die Therapie mit Sulfonylharnstoff wurde unterbrochen und durch Insulin ersetzt, wenn sich der $\mathrm{HbA}_{1 \mathrm{c}}$-Wert nicht unter 6,5\% absenken lies. Endpunkte waren $\mathrm{HbA}_{1 c^{-}}$ Wert, Anteil der Patienten mit $\mathrm{HbA}_{1 \mathrm{c}^{-}}$ Werten von 6,5\% und darunter, Hypoglykämierate und Gewichtsverlauf.

Ergebnisse: Der durchschnittliche $\mathrm{HbA}_{1 \mathrm{c}}{ }^{-}$ Wert war in den 3 Gruppen (Mischinsulin 7,1\%; prandiales Insulin 6,8\% und Insulin Detemir 6,9\%) vergleichbar. Allerdings erreichten mit Mischinsulin weniger Patienten $\mathrm{HbA}_{1 \mathrm{c}}$-Werte von $6,5 \%$ oder darunter $(31,9 \%)$ als mit prandialem Insulin $(44,7 \%)$ oder mit Insulin Detemir (43,2\%). Die durchschnittliche Zahl an Hypoglykämien pro Patientenjahr war am geringsten mit Insulin Detemir (1,7), höher mit Mischinsulin $(3,0)$ und am höchsten mit prandialer Insulintherapie $(5,7$; jeweils $\mathrm{p}<0,001)$.
Das Körpergewicht stieg im Laufe der 3-jährigen Studie um durchschnittlich $5,7 \mathrm{~kg}$ unter Mischinsulin, um $6,4 \mathrm{~kg}$ unter prandialer Insulintherapie und um $3,6 \mathrm{~kg}$ unter Insulin Detemir. Andere Nebenwirkungen waren in den 3 Gruppen vergleichbar häufig.

Folgerung: Bei Typ-2-Diabetiker mit unbefriedigender Stoffwechsellage unter maximal tolerierter Dosis von Metformin und Sulfonylharnstoff wird durch die Kombination mit entweder Mischinsulin, prandialem Insulin oder Insulin Detemir der $\mathrm{HbA}_{1 \mathrm{c}}$-Wert vergleichbar stark gesenkt. Allerdings verfehlen Patienten mit Mischinsulin signifikant häufiger den $\mathrm{HbA}_{1 \mathrm{c}}$-Zielwert von 6,5\%. Unter der Therapie mit Insulin Detemir traten weniger Hypoglykämien auf und es wurde eine geringere Gewichtszunahme berichtet.

Dr. Winfried Keuthage, Münster

Quelle: Holmann RR, Farmer AJ, Davies MJ et al. Three-Year Efficacy of Complex Insulin Regimens in Type 2 Diabetes. N Engl J Med 2009; 361: $1736-1747$ 